Published as: Auer, M. \& Griffiths, M.D. (2013). Behavioral tracking tools, regulation and corporate social responsibility in online gambling. Gaming Law Review and Economics, $17,579-583$.

\title{
Behavioral tracking tools, regulation and corporate social responsibility in online gambling
}

\author{
Michael Auer ${ }^{1}$ and Mark D. Griffiths ${ }^{2}$
}

\author{
neccton ltd., London, UK \\ ${ }^{2}$ Nottingham Trent University, Nottingham, UK
}


Over the last decade, the gambling market has changed dramatically. There are now increased opportunities to gamble almost anywhere and at any time via internet and mobile phone technology. This article briefly focuses on corporate social responsibility in online gambling. Online gambling is regulated differently across the world. For instance, there is no European wide regulation of internet gambling and it is up to individual member states to decide whether they allow online gambling in their territory and whom they grant operating licenses. The same is also true for the USA. There are an increasing number of countries that have legalized online gambling. For example, Denmark has issued more than 30 online gambling licenses. ${ }^{1}$

In addition to state licensed operators (that typically can only offer games to citizens within their jurisdiction), there are large numbers of operators located in places like Malta or Gibraltar that offer their online games to everyone worldwide. Such operators only have to adhere to the responsible gaming standards in one jurisdiction (e.g. Malta, Gibraltar) though they operate globally. Only recently have various states in the European Union regulated their online gaming market. For instance, Italy has entrusted the governmental agency AAMS (Amministrazione Autonoma dei Monopoli Stado - Autonomous Administration of the State Monopolies) with all gaming-related matters. The AAMS is responsible for issuing gaming licenses and for enforcing all legal aspects of gambling. Consequently, Italy has shifted from banning all online gaming into a regulated market where online gambling is offered by private organizations but monitored by a governmental body.

Another example of a partial regulation is currently in progress in Germany. Under a newly ratified law, 20 online sports betting licenses will be issued. ${ }^{2}$ Online casino gambling will still not be allowed under nationwide German 
law. However several federal German states have different laws regarding online gambling and has led to major legal confusion over the past year. Part of this confusion is due to the federal organization of Germany. In 2012, Delaware became the first state to enter legal online casino gambling in the USA. ${ }^{3}$ The legislation allows all types of online games like slots, roulette, blackjack and poker. These recent US developments stand in stark contrast to the Unlawful Internet Gambing Enforcement Act that was signed by George Bush in 2006. ${ }^{4}$ After its introduction it changed the online gambling market as many big operators (e.g., Party Gaming, 888) left the US market.

State licensed operators are not only restricted in who can play their games but must also follow specific responsible gaming procedures as laid down by the jurisdiction in question. Consequently, many operators seek certification by independent organizations such as the World Lottery Organization or GamCare to help in (i) getting operating licenses from regulating gaming authorities, and (ii) demonstrating commitment to social responsibility and facilitating trust amongst its players. In order to be certified by leading accreditation agencies, specific responsible gaming procedures have to be implemented. Such protocols include:

- Age and identify verification: This is to ensure that only adults from within a specific jurisdiction are able to play (i.e., to exclude minors and those living outside the designated country from gambling). Here, responsible online gaming operators use reliable external databases to check the identity of all potential customers.

- Player education: Responsible online gaming operators are now offering players information about the nature of gambling. Such information educates players about different aspects of gambling (e.g., the 
probabilities of winning, potential problems with gambling, false belief systems, the independence of game events, etc.).

- Mandatory and voluntary limit setting: Most state licensed operators now have mandatory cash limits to help players minimize losses. Players cannot exceed certain 'cash in' (deposit) limits or lose more than a certain amount of money within a certain time period (e.g., week or month). Many responsible online gaming operators also offer their players the possibility to voluntarily limit the time and/or money spent gambling.

- 'In-play' notifications: Some responsible online gaming operators also alert their customers about their gambling behavior while they are actually playing. For instance, in order to be certified by GamCare, gaming operators have to implement a 'pop-up' notification system that lets gamblers know they have been playing continuously for an hour.

We recently published a study that investigated the effects of voluntary limit setting among high intensity online gamblers. ${ }^{5}$ We found that limit setting was effective in reducing gambling behavior among the target group (of the most gaming intense players). The effects of the different types of limit setting (i.e., money and time) varied across different game types (for instance, time limits were most effective in reducing intense poker play while spending limits were most effective in reducing intense playing of casino games).

All these responsibility measures are applied to all players but do not take into account the individual gambling behavior. We believe that when it comes to responsible gambling, players should be handled individually, and take into account their individual gambling behavior. Furthermore identification of playing patterns is not enough. In order to change behavior, 
online gaming operators have to communicate with their players. Consequently, behavioral tracking tools (e.g., commercially available tools like mentor (neccton ltd.) and PlayScan (PlayScan AB), or company-developed tools like Observer (888) can help in this regard.

Behavioral tracking tools tend to be customer-centric that supports players' gaming decisions. Some of these tools provide players with personalized information about their gambling behavior. Such systems typically utilize personalized behavioral tracking data in order to give personalized feedback. Here are some of the main reasons why behavioral tracking can be important to online gaming operators.

(1) The psychology of gambling: Players have different motivations for gambling. Some players gamble primarily to relax, whereas other gamblers seek action and excitement. ${ }^{6}$ These, along with several other motivators, lead to specific playing patterns. Behavioral tracking tools can help extract those patterns and advise gamblers accordingly about how they can change their behavior if they so wish.

(2) Motivational self-efficacy enhancement: Studies have shown that messages during or after play have beneficial harm minimization effects on gambling behavior. ${ }^{7-10}$. The best tools should be able to personalize communication with players. Such communication plans should be based on the concept of motivational interviewing, which has proven to be successful in a wide range of areas for instigating behavioral change.

(3) Recovery in the absence of abstinence: Studies have shown that up to $90 \%$ of recovered problem gamblers still occasionally gamble. Behavioral tracking tools should aim to keep gambling safe and fun. ${ }^{11}$ However, 
such systems should also support the small percentage of gamblers who financially (or temporally) overextend themselves.

(4) Identification of high-risk sub-groups: Behavioral tracking tools should be able to identify subgroups of gamblers and provide gamblers with the right information at the right point in time. Furthermore, personalized messages should follow the concept of motivational interviewing to ensure a higher likelihood of behavioral change.

(5) Satisfied players: The main objective of any behavioral tracking tool should be player protection. For most players, gambling is an enjoyable leisure activity. However, a small minority of players can encounter problems. Behavioral tracking tools should be able to detect undesirable behavioral tendencies and initiate personalized communication with players with the aim of preventing problematic gambling. Consequently, players can continue to enjoy their gambling, and customer satisfaction increases.

(6) Increased loyalty: The gaming market - especially the online market - is a highly competitive environment. Attracting new players can be expensive, and every customer lost impacts negatively on the financial investment made through marketing and advertizing. Loyalty is the key to repeat custom over the player's lifetime. The longer that players use an operator's product, the higher the revenue per player. However, once players run into gambling problems, they are typically lost forever. Behavioral tracking tools can help players to enjoy gambling within limits, while extending their lifespan as a player. This gives online gaming operators the opportunity to build up a stable and profitable customer relationship (benefitting both players and operators).

(7) Increased trust: Attracting players in a highly competitive gaming market requires extensive marketing investment. But how can players 
distinguish trustworthy operators? Behavioral tracking tools indicate both objectivity and transparency and help empower trust. It signals a strong customer-centric approach. A high level of trust increases and strengthens existing customer relationships, and helps to attract new customers. Behavioral tracking tools also signal transparency to regulators and the community, which in turn increases confidence in online gaming operators.

Good behavioral tracking tools should be able to support informed player choice, and also help online gaming operators gain more insight into their players' behavioral patterns. Such tools have the potential to supply gaming operators with valuable information through standardized reports. The standardized data interface can be used to immediately answer both ad hoc and recurring questions. This knowledge can in turn be used to optimize the player experience and keep gambling both safe and enjoyable.

The next key issue is to determine which specific features of behavioral tracking tools are the most effective in facilitating and enabling behavioral change in gamblers for the better. The scientific understanding of behavioral tracking tools should (where possible) utilize empirical evidence to underpin their design and implementation. The main target populations for behavioral tracking tools are at-risk players or those who are developing a problem. Behavioral tracking tools should thus focus on significant changes in behavioral indicators such as time spent or money spent. The main focus for behavioral tracking tools lies in providing personalized information and pursuing the motivation to change maladaptive behavior.

For these reasons, it is suggested that behavioral tracking tools should be based on the concepts of the stages of change model. ${ }^{12-13}$. The stages of change model assumes that in order to change people's behavior, a person 
goes through various stages from pre-contemplation through to maintenance. Miller and Rollnick ${ }^{14}$ introduced the related concept of Motivational Interviewing. They found that an emphatic style improved treatment outcomes, regardless of the type of intervention used. One important aspect of these approaches is that they improve the motivation to change by enhancing self-efficacy. Motivational interviewing has proven to be effective in various settings. ${ }^{15-18}$

In gambling environments, the way feedback is presented has proven to be significant. Where as static messages do not seem to be effective, interactive pop-up messages and animated messages appear more effective in changing irrational belief patterns and behavior. ${ }^{7-10,19-20}$ Monoghan and Blaszczynski ${ }^{9}$ demonstrated that self-appraisal messages had a significantly greater selfreported effect on thoughts and behaviors than information and control messages. They also found that messages encouraging self-appraisal resulted in significantly greater effect on self-reported thoughts and behaviors during both the experimental session and in subsequent play of electronic gaming machines. Monaghan and Blaszczynski ${ }^{10}$ also noted that the focus should be on signs that encourage players to reflect on (i) the amount of time or money they have spent, (ii) comparative expenditure patterns to help set personal limits, and (iii) whether they need appropriate self-regulatory action.

In order provide personalized feedback, the individual behavior has to be addressed. Various types of gamblers that have been described in literature can partly be deduced by specific playing patterns, such as binge gamblers who show short but intensive bursts of gambling behavior. ${ }^{21-23}$ Griffiths and Whitty $^{24}$ have also reported that specific behavioral indicators such as chasing losses and tolerance can be detected by behavioral tracking tools. 
Identifying gambling patterns and addressing them with personalized messages that are based on the concept of motivational interviewing is therefore essential in the development of effective behavioral tracking tools. Methodologically, problem gambling can be predicted $^{20}$ but these approaches might not be useful in vivo. Predictive models are complex mathematical formulas that are not transparent to the individual. Different reasons might lead to the classification as a risky gambler. However, changes in behavior can only be achieved if operators provide gamblers personalized feedback about their actual behavior and specific significant changes that might have occurred. For that reason binary predictive models are neither sufficient in helping players to better understand their gambling, nor are they helping to change behavior if necessary.

The findings of Lapham and colleagues ${ }^{25}$ also support this point of view. In their study of web-based alcohol intervention, they suggested that feedback needs to be transparent, as participants are nearly universal in their wish to know how they were assigned to their particular risk category. In offline settings using surveys or clinical interview, players can be classified according to screens such as the South Oaks Gambling Screen ${ }^{6}$, DSM-IV ${ }^{27}$, and/or the Canadian Problem Gambling Index. ${ }^{28}$ However, in real gambling settings, gaming operators only observe players' gambling behavior. Griffiths and Whitty ${ }^{24}$ have described the drawbacks of online behavioral tracking compared to self-reflected information. Using self-excluded players to built predictive models may not be a particularly helpful approach. Selfexclusions can happen for many reasons and are not perfectly correlated with problem gambling. 
To date many online gambling operators have implemented a variety of responsible gaming measures that are helping to keep gambling safe. Age verification processes, player education, mandatory limits as well as the possibility to choose voluntary limits are a few examples of commonly available responsible gaming features. Given the protection that these measures and protocols provide to players, there is perhaps an argument that gaming licenses should only be granted to those companies that have such tools in place (e.g., behavioral tracking software becoming mandatory before a operating license can be granted).

Research has shown that personal feedback is helpful for people in getting gamblers to better understand their behavior and (if necessary) help change. Behavioral tracking opens the door for individual, personalized feedback in online gambling. It could then be used to assign gamblers to specific predescribed groups (e.g., binge gamblers, action-seeking gamblers, or dissociative gamblers) who can then be targeted with appropriate messages. Personalized messages can be developed using the terminology of motivational interviewing. Ultimately, it is important that player feedback is personalized, transparent and motivational, and as research outlined earlier has shown, an entertaining and interactive design appears to correlate with the degree of acceptance by the gambler.

\section{References}

(1) $S_{e e}<\underline{\text { http://www.intergameonline.com/i-gaming/news/8660/denmark- }}$ extends-online-gambling-licences>

(2) $S_{e e}<\underline{\text { http://www.isa-guide.de/isa-gaming/articles/68785.html }>}$ 
(3) See < http://usatoday30.usatoday.com/news/nation/story/2012-0628/delaware-online-gambling/55897914/1>

(4) See $<$ http://calvinayre.com/2012/10/15/legal/unlawful-internetgambling-enforcement-act-2006/>

(5) M. Auer, \& M.D. Griffiths, Limit setting and player choice in most intense online gamblers: An empirical study of online gambling behaviour. JOURNAL OF GAMBLING STUDIES, DOI 10.1007/s10899-012-9332-y (2013)

(6) G. Meyer, T. Hayer, \& M.D. Griffiths, PROBLEM GAMING IN EUROPE: CHALLENGES, PREVENTION, AND INTERVENTIONS. New York: Springer (2009)

(7) S.M. Monaghan, Responsible gambling strategies for Internet gambling: The theoretical and empirical base of using pop-up messages to encourage self-awareness. 25 COMPUTERS IN HUMAN BEHAVIOR. 202-207 (2009).

(8) S.M. Monaghan, \& A. Blaszczynski, Recall of electronic gaming machine signs: A static versus a dynamic mode of presentation, 20 JOURNAL OF GAMBLING ISSUES. 235-267 (2007).

(9) S.M. Monaghan, \& A. Blaszczynski, A, Impact of mode of display and message content of responsible gambling signs for electronic gaming machines on regular gamblers. 26 JOURNAL OF GAMBLING STUDIES. 67-88 (2009). 
(10) S.M. Monaghan, \& A. Blaszczynski, Electronic gaming machine warning messages: information versus self-evaluation. 144 JOURNAL OF PSYCHOLOGY: INTERDISCIPLINARY AND APPLIED. 83-96 (2010).

(11) W.S. Slutske, T.M. Piasecki, A. Blaszczynski, \& N.G. Martin, Pathological gambling recovery in the absence of abstinence. 105 ADDICTION. 2169-2175 (2010).

(12) J.O.P. Prochaska, \& J.M. Prochaska, Why don't people change? Why don't continents move? 9 JOURNAL OF PYSCHOTHERAPY INTEGRATION. 83-102 (1991).

(13) J.O.P. Prochaska, \& C.C. DiClemente, Stages and process of selfchange of smoking: Toward an integrative model of change. 51 JOURNAL OF CONSULTING AND CLINICAL PSYCHOLOGY. 390-395 (1983).

(14) Miller, W.R., \& Rollnick, S. (1991). Motivational interviewing: Preparing People to Change Addictive Behaviour. New York: Guilford Press.

(15) A. Dijkstra, H. De Vries, \& J. Roijackers, Computerized tayloted feedback to change cognitive determinants of smoking: A Dutch field experiment. 13 HEALTH EDUCATION RESEARCH. 197-206 (1998).

(16) K.M. Diskin, \& D. Hodgins, A randomized controlled trial of a single session motivational intervention for concerned gamblers. 47 BEHAVIOUR RESEARCH AND THERAPY. 382-388 (2009)

(17) P. Gooding, \& N. Tarrier, A systematic review and meta-analysis of cognitive-behavioural interventions to reduce problem gambling: Hedging 
our bets? 47 BEHAVIOUR RESEARCH AND THERAPY. 592-607 (2009).

(18) M.K. Steinberg, \& D.M. Ziedonis, J.A. Krejci, \& T.H. Bradon, Motivational interviewing with personalized feedback: a brief intervention for motivating smokers with schizophrenia to seek treatment for tobacco dependence. 4 JOURNAL OF CONSULTING AND CLINICAL PSYCHOLOGY. 732-738 (2004).

(19) M. Cloutier, R. Ladouceur, \& R. Sevigny, Responsible gambling tools: popup messages and pauses on video lottery terminals, 140 JOURNAL OF PSYCHOLOGY: INTERDISCIPLINARY AND APPLIED. 434-438 (2006)

(20) T. Schellink, \& T. Schrans. ATLANTIC LOTTERY CORPORATION VIDEO LOTTERY RESPONSIBLE GAMING FEATURE RESEARCH: FINAL REPORT. Halifax, Nova Scotia: Focal Research Consultants (2002).

(21) M.D. Griffiths, A case study of binge problem gambling. 4 INTERNATIONAL JOURNAL OF MENTAL HEALTH AND ADDICTION. 369-376 (2006).

(22) L. Nower \& A. Blaszczynski, Binge gambling: a neglected concept. 3 INTERNATIONAL JOURNAL OF GAMBLING STUDIES. 23-36 (2003).

(23) A. Sklar, R. Gupta, \& J. Derevensky, Binge gambling behaviours reported by youth in a residential drug treatment setting: A qualitative 
investigation. 22 INTERNATIONAL JOURNAL OF ADOLESCENT MEDICINE AND HEALTH. 153-162 (2010).

(24) M.D. Griffiths, \& M.W. Whitty, Online behavioural tracking in Internet gambling research: ethical and methodological issues. 3 INTERNATIONAL JOURNAL OF INTERNET RESEARCH ETHICS. 104-117 (2010).

(25) G.T. Lapham, E.J. Hawkins, L.J. Chavez, C.E. Achtmeyer, E.C. Williams, R.M. Thomas, E.J. Ludman, K. Kypri, S.C. Hunt, \& K.A. Bradley, Feedback from recently returned veterans on an anonymous web-based brief alcohol intervention. 7 ADDICTION SCIENCE CLINICAL PRACTICE. 7-17 (2012).

(26) H.R. Lesieur, \& S.B. Blume, The South Oaks Gambling Screen (SOGS): A new instrument for the identification of pathological gamblers. 114 AMERICAN JOURNAL OF PSYCHIATRY. 1184-1188 (1987).

(27) American Psychiatric Association, DIAGNOSTIC AND STATISTICAL MANUAL OF MENTAL DISORDERS (4TH ED., TEXT REV.). Washington, DC. (2009)

(28) J. Ferris, \& H. Wynne, (2001). THE CANADIAN PROBLEM GAMBLING INDEX: FINAL REPORT. Ottawa: Canadian Centre on Substance Abuse (2001). 